Ciência Florestal, Santa Maria, v. 22, n. 3, p. 493-503, jul.-set., 2012

ISSN 0103-9954

\title{
SANIDADE, TRANSMISSÃO VIA SEMENTE E PATOGENICIDADE DE FUNGOS EM SEMENTES DE Cedrela fissilis PROCEDENTES DA REGIÃO SUL DO BRASIL
}

\author{
SANITY, TRANSMISSION VIA SEEDS AND FUNGI PATHOGENICITY IN \\ Cedrela fissilis SEEDS FROM THE SOUTH REGION OF BRAZIL
}

\author{
Marília Lazarotto $^{1}$ Marlove Fátima Brião Muniz ${ }^{2}$ Rafael Beltrame ${ }^{3}$ \\ Álvaro Figueiredo dos Santos ${ }^{4}$ Caciara Gonzatto Maciel ${ }^{5}$ Solon Jonas Longhi ${ }^{6}$
}

\begin{abstract}
RESUMO
Pesquisas relacionadas à patologia de sementes de espécies florestais nativas, especialmente aquelas mais aprofundadas, são escassas. Assim, o objetivo deste estudo foi avaliar a sanidade das sementes de cedro através de dois métodos de detecção, avaliar a transmissão de fungos associados às sementes para as plântulas e avaliar a patogenicidade de fungos associados às sementes e transmitidos para as plântulas. Para tanto, sementes de seis procedências, dentre os estados do Rio Grande do Sul, Santa Catarina e Paraná, foram utilizadas. Estas foram submetidas a testes de sanidade, pelos métodos do papel-filtro e batata-dextroseágar (BDA), a teste de transmissão via sementes para as plântulas e a teste de patogenicidade de isolados de Rhizoctonia sp., patógeno escolhido por ser o mais frequente nos testes de sanidade e transmitido via semente. Os fungos encontrados em ambos os testes foram: Pestalotia sp., Rhizoctonia sp., Penicillium sp., Phomopsis sp., Rhizopus sp., Aspergillus sp. e Fusarium sp. Ambos os métodos podem ser utilizados para teste de sanidade em sementes de cedro. Os fungos transmitidos via semente para plântula foram Fusarium sp. e Rhizoctonia sp., sendo que, estes causaram danos nas raízes e posterior tombamento. Foi identificado um isolado de Rhizoctonia sp. patogênico para plântulas de cedro, causando os mesmos sintomas relatados no teste de transmissão.
\end{abstract}

Palavras-chave: cedro; patologia de sementes florestais; Fusarium sp.; Rhizoctonia sp.

\section{ABSTRACT}

Researches about seed pathology of native forest species, especially, those more extensive, are rare. This research aims to evaluate the health of Spanish-cedar seeds through two detection methods, to evaluate the possible transmission of fungi associated to Cedrela fissilis seeds to seedlings and to evaluate the pathogenicity of the fungus transmitted by Cedrela fissilis seeds. For this, seeds from six different locations from Rio Grande do Sul, Santa Catarina and Paraná states were used. The seeds were submitted to sanity tests, by blotter-test and potato-dextrose-agar (PDA) methods, transmission by seeds to the seedlings and to

1. Engenheira Florestal, Msc., Doutoranda do Programa de Pós-graduação em Engenharia Florestal, Centro de Ciências Rurais, Universidade Federal de Santa Maria, Av. Roraima, 1000, CEP 97105-900, Santa Maria (RS). Bolsista CAPES. lilalazarotto@yahoo.com.br

2. Engenheira Agrônoma, Dra., Professora Adjunta do Departamento de Defesa Fitossanitária, Centro de Ciências Rurais, Universidade Federal de Santa Maria, Av. Roraima, 1000, CEP 97105-900, Santa Maria (RS). marlovemuniz@yahoo.com.br

3. Engenheiro Florestal, Msc., Doutorando do Programa de Pós-graduação em Engenharia Florestal, Centro de Ciências Rurais, Universidade Federal de Santa Maria, Av. Roraima, 1000, CEP 97105-900, Santa Maria (RS). Bolsista CNPq. beltrame.rafael@yahoo.com.br

4. Engenheiro Agrônomo, Dr., Pesquisador da Embrapa Florestas, Estrada da Ribeira, km 111, Caixa Postal 319, CEP 83411-000, Colombo (PR). alvaro@cnpf.embrapa.br

5. Engenheira Florestal, Mestranda do Programa de Pós-graduação em Engenharia Florestal, Centro de Ciências Rurais, Universidade Federal de Santa Maria, Av. Roraima, 1000, CEP 97105-900, Santa Maria (RS). Bolsista CAPES. caciaragonzatto@gmail.com

6. Engenheiro Florestal, Dr., Professor Titular Departamento de Ciências Florestais, Centro de Ciências Rurais, Universidade Federal de Santa Maria, Av. Roraima, 1000, CEP 97105-900, Santa Maria (RS). longhi.solon@gmail.com

Recebido para publicação em 30/06/2010 e aceito em 18/07/2011 
the pathogenicity test of Rhizoctonia sp., chosen because it was common in sanity testing and transmitted by seed. The fungi found in both of the methods were: Pestalotia sp., Rhizoctonia sp., Penicillium sp., Phomopsis sp., Rhizopus sp., Aspergillus sp. and Fusarium sp. Both tests can be used for the sanity test in Cedrela fissilis seeds. The fungi transmitted by seeds to the seedlings were Fusarium sp. and Rhizoctonia sp., both causing problems in roots and subsequent damping-off. One of the isolates of Rhizoctonia sp. was identified as pathogenic to seedlings, causing the same symptoms observed on transmission test.

Keywords: Spanish-cedar; pathology of forest seeds; Fusarium sp.; Rhizoctonia sp.

\section{INTRODUÇÃO}

O cedro (Cedrela fissilis Vellozo) é uma espécie florestal de dispersão ampla em toda a Região Sul e Sudeste do Brasil. Considerada uma árvore de bom crescimento, produz madeira de excelente qualidade e tem sido intensamente explorada pelos madeireiros (REITZ et al., 1983). Para Caldas (2006), está entre as espécies prioritárias para a implantação de pomares de sementes, tanto para produção de sementes para restauração ambiental, como exploração econômica, já que esta ocorre em três importantes biomas do Brasil: Cerrado, Mata Atlântica e Pantanal.

Alguns trabalhos, já realizados com a espécie, indicaram os principais patógenos associados às suas sementes, porém, poucos são aqueles em que a preocupação é centrada na transmissão destes via semente, bem como os danos que estes organismos poderão desenvolver em plântulas e mudas no viveiro. Neste contexto, destacam-se trabalhos realizados por Benetti et al. (2009) e Ruiz Filho et al. (2004).

A identificação de fungos associados às sementes pode ser feita por diferentes métodos, sendo os mais comuns os do papel-filtro ou blottertest, e com diferentes composições de meios de cultura. Lucca Filho (1991) cita que, para que um método de análise de sanidade de sementes possa ser considerado satisfatório, ele deve ser econômico no uso do material e equipamento, ser rápido, oferecer segurança e resultados em curto período de tempo. Além disso, seus resultados devem ser reproduzíveis por outros laboratórios dentro de limites estatísticos.

A associação de fungos a sementes de espécies florestais já havia sido mencionada em trabalhos de Gibson (1957), quando o autor verificou a ocorrência de Mucor sp., Aspergillus sp. e Trichoderma sp. em sementes de Pinus patula Mor. Entretanto, segundo Santos et al. (2000), a transmissão de fungos via semente para a plântula ainda é pouco estudada na área florestal do Brasil, diferentemente do que ocorre na Índia, Canadá, Estados Unidos e África, onde as coníferas são as espécies mais estudadas. De modo geral, vários danos podem ser provocados por patógenos, associados às sementes, dentre eles, morte em pré-emergência, podridão radicular, tombamento de mudas, manchas necróticas em folhas e caules, deformações como hipertrofias e subdesenvolvimento, descoloração de tecidos e infecções latentes (NEERGAARD, 1979). Somente o teste de patogenicidade, método relativamente fácil de ser utilizado e que pode ser desenvolvido sem necessidade de equipamentos sofisticados (NECHET e ABREU, 2002), pode indicar se um determinado patógeno causará danos nas mudas da espécie.

Este trabalho teve os seguintes objetivos: avaliar a sanidade das sementes de cedro através de dois métodos de detecção rotineiramente utilizados; avaliar a possível transmissão de fungos associados às sementes de cedro para as mudas; e avaliar a patogenicidade do fungo com maior frequência de incidência nos teste de sanidade e transmitido via sementes de cedro.

\section{MATERIAL E MÉTODOS}

\section{Origem das sementes e local dos experimentos}

As sementes de cedro foram obtidas através de três bancos de sementes: Bolsa de Sementes do Viveiro Florestal da UFSM; Fundação Estadual de Pesquisa Agropecuária - Fepagro Florestas Estação Experimental Boca do Monte; e Banco de Sementes da Embrapa Florestas, totalizando seis amostras de diferentes procedências, como segue na Tabela 1.

As sementes foram coletadas e processadas pelos bancos de sementes de onde foram adquiridas, acondicionadas em saco de papel identificado com espécie, local e data de coleta e armazenadas em câmara fria (temperaturas entre 5 e $10{ }^{\circ} \mathrm{C}$ e baixa umidade relativa), até que fossem enviadas para a realização deste estudo.

Os testes, que serão descritos a seguir, 
TABELA 1: Procedências e datas de coleta de sementes de cedro utilizadas.

TABLE 1: Origins and dates of collection of Cedrela fissilis seeds.

\begin{tabular}{ccc}
\hline Amostra & Procedência & $\begin{array}{c}\text { Data de } \\
\text { coleta }\end{array}$ \\
\hline SM & Santa Maria - RS & $17 / 06 / 2008$ \\
SMO & São Miguel do Oeste - SC & $18 / 06 / 2008$ \\
IRATI & Irati - SC & $16 / 06 / 2008$ \\
CAZUL & Cerro Azul - PR & $21 / 07 / 2008$ \\
LAPA & Contenda da Lapa - PR & $21 / 07 / 2008$ \\
LARGO & Campo Largo - PR & $21 / 07 / 2008$ \\
\hline
\end{tabular}

foram realizados nos Laboratórios de Fitopatologia, de Silvicultura Juarez Martins Hoppe, ambos do Centro de Ciências Rurais - UFSM, e nas instalações da Fepagro Florestas - Estação Experimental de Boca do Monte. Todos os experimentos realizados com avaliação de mudas foram conduzidos em casa de vegetação, com irrigação diária, sem controle de temperatura e umidade. De acordo com Moreno (1961), o clima da região caracterizase como subtropical úmido, do tipo $\mathrm{Cfa}$, segundo a classificação de Köppen, caracterizado por temperatura média anual entre 17,9 e $19,2{ }^{\circ} \mathrm{C}$. As chuvas são bem distribuídas ao longo do ano, sendo que, a precipitação média anual fica em torno de 1.400 a $1.760 \mathrm{~mm}$.

\section{Testes de sanidade}

\section{Em papel-filtro (Blotter-test)}

Foram utilizadas 200 sementes não desinfestadas (REGO, 2008), divididas em quatro repetições, com 50 sementes, distribuídas em caixas de plástico transparente (gerbox), previamente desinfestadas com solução de hipoclorito de sódio $1 \%$, forradas com duas folhas de papel-filtro esterilizadas e umedecidas com água destilada esterilizada. A incubação foi realizada em câmara com temperatura controlada a $22{ }^{\circ} \mathrm{C} \pm 3{ }^{\circ} \mathrm{C}$, com 12 horas de luz branca e 12 horas de escuro, durante sete dias. Em seguida, ocorreu a avaliação e identificação dos fungos.

\section{Em meio batata-dextrose-ágar (BDA)}

As sementes foram desinfestadas em álcool $70 \%$ ( \pm 30 segundos), posteriormente com hipoclorito de sódio (1\%), por um minuto e, após, lavadas em água destilada esterilizada. Após a desinfestação, as sementes foram postas para secar sobre papel-filtro e, em seguida, plaqueadas em meio batata-dextrose-ágar (BDA). Considerando que o meio BDA promove o excessivo crescimento de fungos contaminantes superficiais, a desinfestação superficial é rotineira em testes de sanidade. Para o teste, utilizaram-se 100 sementes divididas em quatro repetições de 25 . A incubação ocorreu em câmara com temperatura de $22^{\circ} \mathrm{C} \pm 3^{\circ} \mathrm{C}$, com 12 horas de luz branca e 12 horas de escuro, por sete dias.

A avaliação, para ambos os testes de sanidade, foi realizada observando-se as estruturas fúngicas, em microscópio estereoscópico e ótico, e a identificação dos fungos foi realizada com o auxílio da literatura especializada (BARNETT e HUNTER, 1972). Os dados da incidência dos fungos foram expressos em percentagem.

\section{Teste de transmissão}

Para cada uma das procedências, foram semeadas 100 sementes não desinfestadas, divididas em quatro repetições de 25 , em bandejas de isopor, com uma semente por célula, usando como substrato vermiculita fina. Não houve incorporação de inóculo de patógeno, a fim de que se verificasse se havia transmissão de patógenos via semente. Essas foram mantidas em casa de vegetação com irrigação diária. Ao final de 35 dias da semeadura, foram realizadas as avaliações, de acordo com metodologia descrita por Rego (2008), quando foi avaliada a emergência de mudas sadias e de mudas com sintomas de doenças. As sementes não germinadas foram retiradas, lavadas em água esterilizada e colocadas em câmara úmida, onde permaneceram por sete dias, para identificação dos patógenos associados a estas e, o mesmo foi feito com as mudas sintomáticas.

\section{Avaliação da patogenicidade de isolados de Rhizoctonia sp. \\ Isolamento dos fungos}

Os isolados de Rhizoctonia spp., foram obtidos nos testes de sanidade, através do isolamento em meio BDA e sulfato de estreptomicina. Somente este gênero foi escolhido, por ter ocorrido na maioria das procedências e ter sido transmitido com maior frequência. Os isolados de Fusarium sp. não foram utilizados neste momento, pois estavam presentes apenas em duas procedências e serão aproveitados em pesquisas futuras.

Para que houvesse garantia de pureza dos fungos isolados, foi realizada a repicagem com apenas uma unidade formadora de colônia (UFC) 
que, no caso de Rhizoctonia sp., foi um pequeno fragmento de hifa retirado de placas de meio ágar-água (AA) - e transferido para meio batatadextrose-ágar (BDA), baseando-se na metodologia descrita por Fernandes (1993). Assim que o fungo ocupou toda a placa, aos dez dias da repicagem, foi realizada a inoculação em sementes. Quatro isolados foram utilizados: $\mathrm{R}_{1}$ - Campo Largo - PR, obtido no teste de sanidade em BDA, $\mathrm{R}_{5}-$ Cerro Azul - PR, $\mathrm{R}_{6}$ - Irati - SC - BDA e $\mathrm{R}_{8}$ - Santa Maria - RS - BDA.

\section{Inoculação}

Após o período de incubação (dez dias sob temperatura de $22{ }^{\circ} \mathrm{C} \pm 3{ }^{\circ} \mathrm{C}$, com 12 horas de luz branca e 12 horas de escuro), foi realizada a inoculação dos fungos nas sementes através do método de contato com a cultura fúngica, conforme Rego (2008). Somente uma amostra de sementes, Cerro Azul - PR, foi utilizada. Esta amostra foi escolhida por apresentar, nos testes iniciais, resultados satisfatórios referentes ao vigor. Com este procedimento, evitou-se que houvesse confusão entre os sintomas causados pelo patógeno, com aspectos de baixa qualidade fisiológica de sementes e das mudas resultantes. Anteriormente à inoculação, as sementes foram desinfestadas com álcool $70 \%$ (30 segundos) e hipoclorito de sódio $1 \%$ ( 1 minuto), lavadas emágua destilada esterilizada e deixadas secar sob papel-filtro esterilizado. Posteriormente, as sementes foram colocadas em contato com as culturas fúngicas crescidas em placas de Petri contendo BDA, e as sementes testemunhas, apenas em meio BDA. Estas foram mantidas, durante 24 horas, em laboratório, sem condições controladas de temperatura e umidade. Após este período, estas sementes foram semeadas em bandejas de isopor (sementeira), previamente esterilizadas com hipoclorito de sódio $1 \%$, durante 24 horas, contendo vermiculita fina. Foram semeadas quatro repetições de 25 sementes para cada isolado testado.

\section{Avaliações}

Foram realizadas avaliações semanais, a partir do $14^{\circ}$ dia até o $60^{\circ}$ dia, contando o número de mudas emergidas e número total de mudas com sintomas, devido à presença do fungo. As mudas sintomáticas foram lavadas em água esterilizada, a seguir deixadas para secar sobre papel-filtro esterilizado e, posteriormente, colocadas em placas de Petri, contendo BDA, e mantidas em câmara de incubação a uma temperatura de $20^{\circ} \mathrm{C}$, durante uma semana, para o reisolamento do fungo.

\section{Procedimento estatístico}

$\mathrm{O}$ delineamento experimental utilizado foi completamente casualizado, com quatro repetições para cada teste realizado. Para a análise de variância, os dados obtidos em percentagem foram transformados segundo arc sen $\sqrt{ }(\mathrm{x} / 100)$. A comparação das médias foi feita pelo teste de Tukey a $5 \%$ de probabilidade de erro, utilizando-se o software Sistema de Análise Estatística - SANEST (ZONTA e MACHADO, 1986).

\section{RESULTADOS E DISCUSSÃO}

\section{Sanidade}

A partir dos dados levantados, verificou-se que Pestalotia sp. e Phomopsis sp. ocorreram em todas as procedências, Ascochyta sp. e Rhizoctonia sp. ocorreram em todas as procedências, exceto em São Miguel do Oeste - SMO, porém, esta amostra obteve alta incidência de Fusarium sp., como pode ser observado na Tabela 2.

Além dos fungos mencionados na Tabela 2, outros ocorreram em menores incidências, tais como Alternaria sp., Aspergillus sp., Epicoccum sp., Rhizopus sp., Sphaeropsis sp. e Trichoderma sp., porém, em poucas amostras e em baixas incidências. A percentagem de sementes sadias, isto é, isentas de estruturas fúngicas associadas, também foi considerável em algumas amostras, como em Contenda da Lapa - LAPA (17 \%), Santa Maria SM (15,5 \%) e Campo Largo - LARGO (11,5 \%), para as demais amostras, as percentagens foram inferiores a $5 \%$.

Na Tabela 3, observa-se a incidência de fungos em sementes de cedro, através do método de incubação em meio batata-dextrose-ágar (BDA). Penicillium sp. e Rhizoctonia sp. ocorreram em todas as procedências testadas, Aspergillus sp. e Pestalotia sp. ocorreram em todas as amostras, exceto em IRATI e SMO, respectivamente. Além dos fungos apresentados na Tabela 3, Fusarium sp. também ocorreu, porém, apenas na amostra de São Miguel do Oeste - SMO, com incidência de $7 \%$.

Cherobini et al. (2008), utilizando o teste de sanidade em papel-filtro, encontraram, associados às sementes de cedro, somente os fungos: Aspergillus spp., Chaetomium spp., Penicillium spp. e Trichoderma spp.; já Mieth et al. (2007), encontraram Fusarium spp., Penicillium spp., 
Aspergillus spp., Verticillium spp., Rhizoctonia spp.

Para Christensen (1973), Aspergillus sp. e Penicillium sp. são considerados fungos de armazenamento, pois a incidência pode aumentar no período pós-colheita. Segundo Oliveira et al. (1997), estes fungos, quando associados às sementes de milho, além de terem sua incidência maximizada com o período de armazenamento, podem causar redução no percentual de germinação. Segundo Ferreira (1989), algumas espécies de Fusarium têm sido relatadas, causando tombamento em pré ou pós-emergência de plântulas de espécies florestais, sendo problema comum em sementes. Para Dhingra et al. (1980) e Machado (1988), as associações com fungos do gênero Fusarium, em sementes de culturas agronômicas, ocorrem durante a formação ou maturação do fruto e, cuidados na colheita e no manuseio, podem reduzi-las.

Fungos do gênero Rhizoctonia são conhecidos por causar a queima de folhas e mela de estacas em eucalipto (SILVEIRA et al., 2000) e já existem relatos para outras espécies florestais, tais como podridão-de-raízes em erva-mate (Ilex paraguariensis A. St.-Hil) (POLETTO et al., 2007). Phomopsis spp. também é comumente encontrado em sementes de espécies florestais e pode ocasionar doenças em plantas adultas, como foi relatado por Anjos et al. (2001), quando este fungo causou queima das folhas em aroeira (Myracrodruon urundeuva Allem.) no Distrito Federal.

TABELA 2: Incidência de fungos (\%), detectados pelo teste em papel-filtro, associados às sementes de cedro coletadas de seis procedências.

TABLE 2: Fungi incidence (\%), detected by blotter-test, associated with Cedrela fissilis seeds from six different origins.

\begin{tabular}{cccccccc}
\hline \multirow{2}{*}{ Procedências s } & \multicolumn{7}{c}{ Fungos } \\
\cline { 2 - 7 } & Ascochyta sp. & Colletotrichum sp. & Fusarium sp. & Penicillium sp. & Pestalotia sp. & Phomopsis sp. & Rhizoctonia sp. \\
\hline SM & $28 \mathrm{ab}$ & $18,5 \mathrm{a}$ & $0 \mathrm{~d}$ & $5,5 \mathrm{c}$ & $4,5 \mathrm{e}$ & $1 \mathrm{e}$ & $32 \mathrm{ab}$ \\
$\mathrm{SMO}$ & $0 \mathrm{~d}$ & $19,5 \mathrm{a}$ & $41,5 \mathrm{a}$ & $16 \mathrm{~b}$ & $3,5 \mathrm{e}$ & $19 \mathrm{~b}$ & $0 \mathrm{~d}$ \\
$\mathrm{IRATI}$ & $2 \mathrm{c}$ & $21 \mathrm{a}$ & $9,5 \mathrm{~b}$ & $28 \mathrm{a}$ & $10 \mathrm{~d}$ & $15 \mathrm{bc}$ & $20,5 \mathrm{c}$ \\
CAZUL & $22,5 \mathrm{~b}$ & $0 \mathrm{~d}$ & $1,5 \mathrm{c}$ & $1 \mathrm{~d}$ & $25 \mathrm{~b}$ & $26,5 \mathrm{ab}$ & $28,5 \mathrm{~b}$ \\
LAPA & $20 \mathrm{~b}$ & $4,5 \mathrm{c}$ & $0 \mathrm{~d}$ & $0 \mathrm{~d}$ & $38,5 \mathrm{a}$ & $3,5 \mathrm{~d}$ & $21 \mathrm{c}$ \\
LARGO & $30,5 \mathrm{a}$ & $7 \mathrm{~b}$ & $0 \mathrm{~d}$ & $0 \mathrm{~d}$ & $16,5 \mathrm{c}$ & $30,5 \mathrm{a}$ & $37,5 \mathrm{a}$ \\
\hline CV & 27,6 & 22,1 & 14,5 & 15,2 & 15,3 & 28,2 & 14,9 \\
\hline
\end{tabular}

Em que: SM: Santa Maria-RS; SMO: São Miguel do Oeste-SC; IRATI: Irati-SC; CAZUL: Cerro Azul-PR; LAPA: Contenda da Lapa-PR; LARGO: Campo Largo-PR. Médias seguidas por mesma letra na coluna não diferem entre si pelo teste de Tukey ao nível de $5 \%$ de probabilidade de erro.

TABELA 3: Incidência de fungos (\%), detectados pelo método de incubação em meio BDA, associados às sementes de cedro coletadas em seis procedências.

TABLE 3: Fungi incidence (\%), detected by PBA (potato-dextrose-agar), associated with Cedrela fissilis seeds from six different origins.

\begin{tabular}{cccccccc}
\hline \multirow{2}{*}{ Procedências } & \multicolumn{7}{c}{ Fungos } \\
\cline { 2 - 8 } & Aspergillus sp. & Penicillium sp. & Pestalotia sp & Phomopsis sp. & Rhizoctonia sp. & Rhizopus sp. & Trichoderma sp. \\
\hline SM & $1 \mathrm{~b}$ & $48 \mathrm{a}$ & $1 \mathrm{~d}$ & $0 \mathrm{c}$ & $64,5 \mathrm{a}$ & $0 \mathrm{c}$ & $0 \mathrm{~b}$ \\
SMO & $20 \mathrm{a}$ & $42 \mathrm{a}$ & $0 \mathrm{~d}$ & $0 \mathrm{c}$ & $5 \mathrm{e}$ & $13 \mathrm{a}$ & $18 \mathrm{a}$ \\
IRATI & $0 \mathrm{~b}$ & $23 \mathrm{~b}$ & $13 \mathrm{c}$ & $2 \mathrm{~b}$ & $46 \mathrm{c}$ & $2 \mathrm{bc}$ & $0 \mathrm{~b}$ \\
CAZUL & $3 \mathrm{~b}$ & $14 \mathrm{c}$ & $23 \mathrm{~b}$ & $17 \mathrm{a}$ & $53 \mathrm{~b}$ & $0 \mathrm{c}$ & $3 \mathrm{~b}$ \\
LAPA & $3 \mathrm{~b}$ & $23 \mathrm{~b}$ & $30 \mathrm{a}$ & $0 \mathrm{c}$ & $26 \mathrm{~d}$ & $11 \mathrm{ab}$ & $0 \mathrm{~b}$ \\
LARGO & $2 \mathrm{~b}$ & $10 \mathrm{~d}$ & $19 \mathrm{bc}$ & $19 \mathrm{a}$ & $53 \mathrm{~b}$ & $0 \mathrm{c}$ & $0 \mathrm{~b}$ \\
\hline CV & 25,3 & 26,8 & 27,7 & 20,9 & 22,0 & 28,9 & 19,6 \\
\hline
\end{tabular}

Em que: SM: Santa Maria-RS; SMO: São Miguel do Oeste-SC; IRATI: Irati-SC; CAZUL: Cerro Azul-PR; LAPA: Contenda da Lapa-PR; LARGO: Campo Largo-PR. Médias seguidas por mesma letra na coluna não diferem entre si pelo teste de Tukey ao nível de $5 \%$ de probabilidade de erro. 
Observando-se os dois métodos de detecção utilizados, papel-filtro e BDA, observa-se que Rhizoctonia sp. e Pestalotia sp. foram os fungos que ocorreram no maior número de amostras e em ambos os métodos testados (Tabela 4). Penicillium sp. ocorreu, coincidentemente, em quatro amostras, nos métodos de detecção testados.

O método do papel-filtro conseguiu detectar a maior quantidade de fungos; já o meio BDA não foi eficiente na detecção de Ascochyta sp., Colletotrichum sp., Epicoccum sp. e Sphaeropsis sp. Estes fungos poderiam estar colonizando as sementes, somente superficialmente, sendo eliminados pela desinfestação utilizada pelo método em BDA. Fusarium sp. foi observado em alta incidência na amostra SMO em papel-filtro (Tabela 2), já em BDA teve sua incidência drasticamente reduzida (Tabela 3 ).

Rhizoctonia spp., além de ser um dos fungos com maior número de detecções coincidentes entre ambos os métodos testados, também obteve a maior incidência média para o papel-filtro e meio BDA, indicando uma grande contaminação das sementes por este patógeno.

O método de papel-filtro detectou uma maior quantidade de fungos, porém, este pode ter sido favorecido pela ausência de assepsia superficial das sementes. A desinfestação das sementes para a realização do plaqueamento em BDA é imprescindível, pois, na ausência desta, os fungos que estão contaminando superficialmente as sementes impossibilitariam a avaliação e o teste seria perdido, pois este meio de cultura é muito rico em nutrientes, o que não ocorre em papel-filtro. Portanto, pode-se considerar que os dois métodos testados foram eficientes na detecção da maior parte dos fungos encontrados nas sementes, como também verificado por Ruiz Filho et al. (2004), onde constataram que, tanto o método de detecção em papel-filtro quanto em meio $\mathrm{BDA}$, foram eficientes na detecção dos fungos em sementes de cedro (Cedrela fissilis); além disso, os mesmos autores encontraram os seguintes gêneros de fungos: Phomopsis, Phoma, Cladosporium, Fusarium, Curvularia, Pestalotia, Penicillium, Aspergillus, Trichoderma, Rhizopus, Chaetomium, Ascochyta e Stilbum. Benetti et al. (2009) encontraram alguns fungos potencialmente fitopatogênicos em papelfiltro e BDA, como Fusarium spp., Phomopsis spp., Colletotrichum spp., Machophomina spp.,

TABELA 4: Número de amostras de sementes de cedro em que houve ocorrência de fungos em papelfiltro (PF) e batata-dextrose-ágar (BDA), números de amostras em que houve coincidência na detecção de fungos nos dois métodos utilizados e incidência média (\%) de fungos.

TABLE 4: Number of seed samples of Cedrela fissilis that was occurrence of fungi on blotter-test (PF) and potato dextrose agar (BDA), numbers of samplings which there was agreement in the detection of fungi in the two methods used and the mean incidence (\%) of fungi.

\begin{tabular}{lccccc}
\hline & \multicolumn{2}{c}{ Ocorrência $^{1}$} & & \multicolumn{2}{c}{ Incidência Média $^{1}(\%)$} \\
\hline \multicolumn{1}{c}{ Fungos } & PF & BDA & Coincidência $^{2}$ & PF & BDA \\
\hline Ascochyta sp. & 5 & 0 & 0 & 20,6 & 0 \\
Aspergillus sp. & 2 & 5 & 2 & 1,5 & 5,8 \\
Colletotrichum sp. & 5 & 0 & 0 & 14,1 & 0 \\
Epicoccum sp. & 1 & 0 & 0 & 1,0 & 0 \\
Penicillium sp. & 4 & 6 & 4 & 12,6 & 26,7 \\
Pestalotia sp. & 6 & 5 & 5 & 16,3 & 17,2 \\
Rhizoctonia sp. & 5 & 6 & 5 & 27,9 & 41,3 \\
Trichoderma sp. & 3 & 2 & 0 & 1,2 & 10,5 \\
Phomopsis sp. & 5 & 3 & 3 & 19,1 & 12,7 \\
Fusarium sp. & 3 & 1 & 1 & 17,5 & 7,0 \\
Rhizopus sp. & 4 & 3 & 3 & 3,7 & 8,7 \\
Sphaeropsis sp. & 1 & 0 & 0 & 2,5 & 0 \\
\hline Média & - & - & - & 11,5 & 16,2 \\
Desvio padrão & & & & 9,2 & 12,1 \\
\hline
\end{tabular}

Em que: ${ }^{1}$ Número de amostras avaliadas $=6 ;{ }^{2}$ Número de vezes em que houve coincidência na detecção de fungos entre os dois métodos. 
Pestalotia spp. e Cladosporium spp. além de fungos considerados de armazenamento, como Penicillium spp., Chaetomiun spp., Aspergillus spp., Rhizopus spp., Trichotecium spp., Epiccocum spp.

Esses métodos também foram comparados para outras espécies florestais, como nos trabalhos realizados por Medeiros et al. (1992), em sementes de aroeira (Astronium urundeuva Engl.), observando que a eficiência dos métodos foi diferente para cada fungo. Porém, o método de papel de filtro a $20^{\circ} \mathrm{C}$ foi sugerido por eles, pois detectou a maior variedade de fungos. Já para Magalhães et al. (2008), testando os mesmos métodos utilizados no presente estudo para detecção de fungos em sementes de coquinhoazedo (Butia capitata Becc.), verificaram que o teste em BDA foi mais eficiente.

Apesar de ambos os métodos utilizados serem eficientes na detecção de fungos em sementes de cedro, como relatado por vários autores e verificado no presente estudo, Gasparotto et al. (2009), afirmam que o teste de sanidade em papelfiltro é eficaz para detectar a maioria de fungos veiculados por sementes e ainda destacam que é uma das técnicas mais simples e baratas para este fim.

\section{Transmissão}

Os resultados do teste de transmissão, apresentados a seguir (Tabela 5), complementam os testes de detecção, pois comprovam se os fungos presentes nas sementes são transmitidos para as mudas e, em caso positivo, os tipos de sintomas que produzirão. Verificou-se que a amostra procedente de São Miguel do Oeste (SC) - SMO obteve a maior média para sementes não germinadas (SNG), bem como a menor média para mudas normais $(\mathrm{MN})$, como pode ser visto na Tabela 5. Esta amostra também obteve a segunda maior média de mudas sintomáticas, as quais apresentaram sintomas de apodrecimento das raízes, colo e cotilédones (Figura 1), causados por Fusarium sp., o mesmo ocorrendo para a amostra de Irati (SC) - IRATI. Ambas as amostras apresentavam associação de Fusarium sp. em suas sementes, detectado no teste de papel-filtro, como já visto na Tabela 2 .

A amostra CAZUL, procedente de Cerro Azul (PR), apresentou a maior média (78 \%) para mudas normais, seguida de SM (72\%) e LARGO (68\%), e baixa percentagem de mudas sintomáticas. Já SM, de procedência de Santa Maria (RS), obteve a maior percentagem de mudas sintomáticas (25\%), as quais apresentaram sintomas de apodrecimento do colo e raízes causado por Rhizoctonia sp. (Figura 1) As duas procedências restantes também apresentaram mudas infectadas por Rhizoctonia sp. Algumas não apresentavam sintomas visíveis no colo ou parte aérea, contudo, quando foram arrancadas, encontravam-se com raízes escurecidas e fracas devido à associação com Rhizoctonia sp.

Entre as sementes não germinadas, verificou-se a presença de alguns fungos, já presentes nos testes de detecção, que podem ter afetado a emergência, ou mesmo terem sido transmitidos para as mudas (Tabela 6).

TABELA 5: Porcentagem média de sementes não germinadas (SNG), mudas normais (MN), mudas sintomáticas (MS) e fungos encontrados em mudas sintomáticas no teste de transmissão com sementes de cedro de diferentes procedências.

TABLE 5: Average percentage of non-germinated seeds (SNG), normal seedlings (PN), symptomatic seedlings (PS) and fungi found on symptomatic seedlings in transmission test with Cedrela fissilis seeds from different origins.

\begin{tabular}{ccccc}
\hline Procedências & SNG & MN & MS & Fungos \\
\hline SM & $3 \mathrm{c}$ & $72 \mathrm{ab}$ & $25 \mathrm{a}$ & Rhizoctonia $\mathrm{sp}$. \\
SMO & $61 \mathrm{a}$ & $23 \mathrm{c}$ & $16 \mathrm{ab}$ & Fusarium $\mathrm{sp}$. \\
IRATI & $39 \mathrm{~b}$ & $57 \mathrm{~b}$ & $4 \mathrm{c}$ & Fusarium sp. \\
CAZUL & $17 \mathrm{c}$ & $78 \mathrm{a}$ & $5 \mathrm{bc}$ & Rhizoctonia sp. \\
LAPA & $43 \mathrm{ab}$ & $47 \mathrm{~b}$ & $10 \mathrm{bc}$ & Rhizoctonia $\mathrm{sp}$. \\
LARGO & $24 \mathrm{bc}$ & $68 \mathrm{ab}$ & $8 \mathrm{bc}$ & Rhizoctonia sp. \\
\hline CV $(\%)$ & 16,4 & 11,7 & 28,3 & - \\
\hline
\end{tabular}

Em que: SM: Santa Maria-RS; SMO: São Miguel do Oeste-SC; IRATI: Irati-SC; CAZUL: Cerro Azul-PR; LAPA: Contenda da Lapa-PR; LARGO: Campo Largo-PR. Médias seguidas por mesma letra na coluna não diferem entre si pelo teste de Tukey ao nível de $5 \%$ de probabilidade de erro. 
Fusarium sp. apareceu em alta incidência, 60 e $64 \%$ nas amostras SMO e IRATI, respectivamente, as quais também apresentaram mudas infectadas por este fungo no teste de transmissão (Tabela 7). Penicillium sp. apareceu em algumas amostras, sendo sua incidência superior (20\%) em SMO, amostra que também obteve a maior média para sementes não germinadas (Tabela 5), podendo ser este fungo um dos responsáveis pela perda da viabilidade das sementes desta amostra. Phomopsis sp. também foi detectado nas sementes não germinadas (Tabela 6), mas não foi transmitido para mudas, indicando a ausência de transmissão via sementes.

Outros fungos, além dos apresentados na Tabela 6, foram detectados nas sementes não germinadas, mas em menores incidências e somente em algumas amostras, tais como: Ascochyta sp. nas
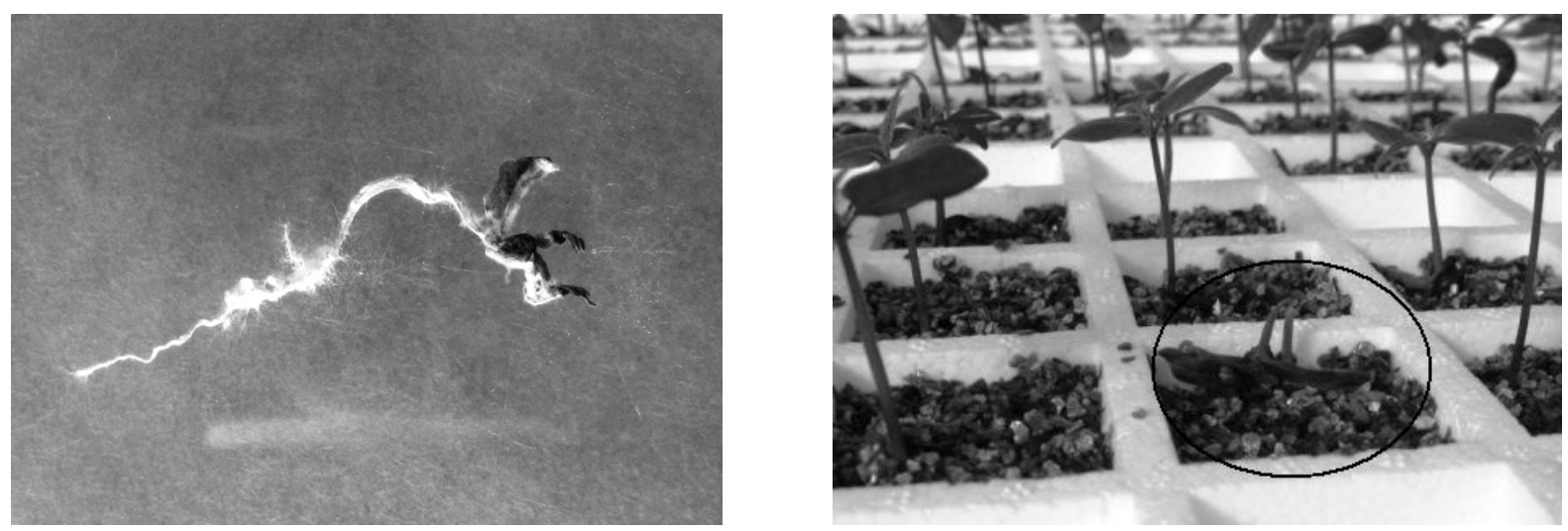

FIGURA 1: Sintomas de tombamento causados por Fusarium sp. (A) e Rhizoctonia sp. (B).

FIGURE 1: Symptoms of damping-off caused by Fusarium sp. (A) and Rhizoctonia sp. (B).

TABELA 6: Incidência (\%) dos principais fungos encontrados nas sementes não germinadas no teste de transmissão em sementes de cedro de diferentes procedências.

TABLE 6: Incidence (\%) of the principal fungi found in non-germinated seeds in the transmission test on Cedrela fissilis seeds from different origins.

\begin{tabular}{cccc}
\hline \multirow{2}{*}{ Procedências } & \multicolumn{3}{c}{ Fungos } \\
\cline { 2 - 4 } & Fusarium sp. & Penicillium sp. & Phomopsis sp. \\
\hline SM & $12 \mathrm{~b}$ & $8 \mathrm{a}$ & $36 \mathrm{~b}$ \\
SMO & $60 \mathrm{a}$ & $20 \mathrm{a}$ & $20 \mathrm{~b}$ \\
IRATI & $64 \mathrm{a}$ & $8 \mathrm{a}$ & $28 \mathrm{~b}$ \\
CAZUL & $0 \mathrm{c}$ & $0 \mathrm{~b}$ & $64 \mathrm{a}$ \\
LAPA & $36 \mathrm{ab}$ & $0 \mathrm{~b}$ & $24 \mathrm{~b}$ \\
LARGO & $12 \mathrm{~b}$ & $12 \mathrm{a}$ & $36 \mathrm{~b}$ \\
\hline CV $(\%)$ & 25,4 & 35,7 & 18,8 \\
\hline
\end{tabular}

Em que: SM: Santa Maria-RS; SMO: São Miguel do Oeste-SC; IRATI: Irati-SC; CAZUL: Cerro Azul-PR; LAPA: Contenda da Lapa-PR; LARGO: Campo Largo-PR. Médias seguidas por mesma letra na linha não diferem entre si pelo teste de Tukey ao nível de $5 \%$ de probabilidade de erro.

Ci. Fl., v. 22, n. 3, jul.-set., 2012 
uma alta percentagem de transmissão $(42,8 \%)$, já Rhizoctonia solani não foi transmitida através de sementes desta espécie.

O gênero Rhizoctonia pertence a um grupo de espécies fúngicas que sobrevivem saprofiticamente no solo, infectando plantas nativas, ou em estádio de dormência, na forma de micélio e escleródios (CASTRO, 2007) e, até o presente estudo, não foram encontrados relatos sobre possível transmissão deste através de sementes de espécies florestais.

Poucos trabalhos com transmissão de fungos em sementes florestais foram realizados, entre eles, pode-se citar o de Lisboa-Padulla (2009) com resultados interessantes, onde foi verificada a transmissão de Pestalotiopsis sp. e Cladosporium cladosporioides de sementes para plântulas de paubrasil (Caesalpinia echinata Lam.), associados a sintomas de lesões no cotilédone.

\section{Patogenicidade}

Arealização de teste de patogenicidade pode confirmar ou excluir a hipótese de que os fungos encontrados associados às sementes e transmitidos por elas são mesmo patogênicos à espécie florestal em estudo. Na Tabela 7, encontram-se os resultados do teste de patogenicidade de isolados de Rhizoctonia sp. em mudas de cedro, inoculados via semente.

Com relação à emergência de mudas, não houve diferenças significativas entre os isolados testados, entretanto, a maior percentagem de mudas sintomáticas foi observada na inoculação com o isolado $\mathrm{R}_{1}$, sendo que, somente este, diferiu da testemunha. Desta forma, apenas o isolado $R_{1}$ foi considerado patogênico, pois a diferença estatística em relação à testemunha demonstra que os sintomas ocorreram por efeito da inoculação. A ocorrência de mudas sintomáticas na testemunha refere-se à contaminação natural das sementes por Rhizoctonia $\mathrm{sp}$., como pode ser visto nos resultados nos testes de sanidade (Tabelas 2, 3 e 4).

Os sintomas observados nas mudas foram manchas ou queima nos cotilédones e primeiras folhas verdadeiras, apodrecimento do colo, seguido de tombamento destas. Quando não ocorriam sintomas aparentes, muitas vezes, as raízes encontravam-se escurecidas e enfraquecidas, resultado da associação deste patógeno com os tecidos da radícula, sendo este, o primeiro relato de patogenicidade de Rhizoctonia sp. para a espécie florestal Cedrela fissilis.

Benetti et al. (2009) testaram a patogenicidade de quatro isolados de Fusarium spp. e um de Pestalotia sp., sendo que, três dos isolados de Fusarium spp., ocasionaram baixa emergência de plântulas de cedro. Santos et al. (1992), verificaram a patogenicidade de Fusarium oxysporum e Rhizoctonia solani provenientes de sementes de urucum (Bixa orellana L.) em mudas da espécie, e verificaram que ambos os patógenos causaram damping-off. Tal como neste último trabalho citado, Rhizoctonia também se mostrou patogênica em mudas de cedro, causando tombamento.

Mendes et al. (2005) testaram a patogenicidade de Phomopsis sp., Colletotrichum gloeosporioides, Fusarium solani e Pestalotiopsis sp. oriundos de sementes de sabiá (Mimosa caesalpiniaefolia) em mudas da espécie, onde somente os dois últimos foram patogênicos, causando murcha e manchas foliares diminutas de coloração branco-acinzentada, respectivamente. Ferreira (1989) ressalta que fungos do gênero Rhizoctonia, mesmo na floresta, possuem uma ampla gama de hospedeiros, onde se tornam responsáveis pela podridão de raízes, lesões em hastes, tombamento de mudas, manchas foliares, mela e queima de folhas.

TABELA 7: Testes de patogenicidade com inoculação de isolados de Rhizoctonia sp. em sementes de cedro: porcentagem de emergência e de mudas sintomáticas (MS).

TABLE 7: Pathogenicity test with inoculation of Rhizoctonia sp. in Cedrela fissilis seeds: emergence percentage and symptomatic seedlings (MS).

\begin{tabular}{ccccccc}
\hline \multirow{2}{*}{ Variáveis } & $\mathrm{R}_{1}$ & $\mathrm{R}_{5}$ & $\mathrm{R}_{6}$ & $\mathrm{R}_{8}$ & Testemunha & CV (\%) \\
\cline { 2 - 6 } & $92 \mathrm{a}$ & $91 \mathrm{a}$ & $93 \mathrm{a}$ & $94 \mathrm{a}$ & $96 \mathrm{a}$ & 6,0 \\
\hline Emergência (\%) & $23 \mathrm{a}$ & $9 \mathrm{~b}$ & $9 \mathrm{~b}$ & $6 \mathrm{~b}$ & $4 \mathrm{~b}$ & 23,2 \\
MS (\%) & &
\end{tabular}

Em que: Médias seguidas por mesma letra na linha não diferem entre si pelo teste de Tukey ao nível de $5 \%$ de probabilidade de erro. $\mathrm{R}_{1}, \mathrm{R}_{5}, \mathrm{R}_{6}, \mathrm{R}_{8}$ : isolados de Rhizoctonia $\mathrm{sp}$. 


\section{CONCLUSÕES}

Os métodos para condução do teste de sanidade, papel-filtro e meio batata-dextrose-ágar, são adequados para a realização de detecção de fungos em sementes de cedro;

Fusarium sp. e Rhizoctonia sp. são transmitidos para as plântulas via sementes, causando problemas radiculares e tombamento de plântulas;

Rhizoctonia sp. é patogênico para cedro, causando manchas ou queima nos cotilédones e primeiras folhas verdadeiras, apodrecimento do colo, seguido de tombamento da plântula pela associação do patógeno com as raízes.

\section{AGRADECIMENTOS}

À Coordenação de Aperfeiçoamento de Pessoal de Nível Superior (CAPES) pela concessão da bolsa de mestrado; à Embrapa Florestas - Colombo/PR e à Bolsa de Sementes do Viveiro Florestal da UFSM, pelo fornecimento das sementes de cedro; e à Fepagro Florestas - Estação Experimental de Boca do Monte - Santa Maria/ RS, pela disponibilização de sua infraestrutura para realização de parte deste estudo.

\section{REFERÊNCIAS BIBLIOGRÁFICAS}

ANJOS, J. R. N. et al. Ocorrência de queima das folhas causada por Phomopsis sp. em aroeira no Distrito Federal. Fitopatologia Brasileira, Brasília, v. 26, n. 3, p. 549-650, set. 2001.

BALARDIN, C. R. et al. Possibilidade de transmissão de Fusarium solani f.sp. glycines, agente causal da podridão vermelha da raiz da soja, através da semente. Fitopatologia Brasileira, Brasília, v. 30, n. 6, p. 574-581, nov./dez. 2005.

BARNETT, H. L.; HUNTER, B. B. Illustrated genera of imperfect fungi. 3rd ed. Minnesota: Burgess Publishing Company, 1972.

BENETTI, S. C. et al. Levantamento de fungos em sementes de cedro e avaliação da patogenicidade de Fusarium sp. e Pestalotia sp. Pesquisa Florestal Brasileira, Colombo, n. 58, p. 79-83, jan./jun. 2009. CALDAS, L. S. Pomares de sementes de espécies nativas as funções das redes de sementes. In: HIGA, A. R.; SILVA, L. D. Pomar de sementes de espécies florestais nativas. Curitiba: FUPEF do Paraná, 2006. p. 227-241.

CASTRO, C. V. B. Caracterização morfológica e molecular de isolados de Rhizoctonia solani Kuhn. 2007. 67 f. Dissertação (Mestrado em Agronomia)Universidade Federal Rural da Amazônia, Belém, 2007.

CHEROBINI, E. A. I.; MUNIZ, M. F. B.; BLUME, E. Avaliação da qualidade de sementes e mudas de cedro. Ciência Florestal, Santa Maria, v. 18, n. 1, p. 65-73, jan./mar. 2008.

CHRISTENSEN, C. M. Loss of viability in storage microflora. Seed Science and Technology, Zurich, v. 1, n. 3, p. 547-562, 1973.

DHINGRA, O. D.; MUCHOVEJ, J. J.; CRUZ FILHO, J. Tratamento de sementes: controle de patógenos. Viçosa: UFV, 1980. 121 p.

FERNANDES, M. R. Manual para laboratório de fitopatologia. Passo Fundo: EMATER - CNPT, 1993. $128 \mathrm{p}$.

FERREIRA, F. A. Patologia florestal: principais doenças florestais no Brasil. Viçosa: UFV, SIF, 1989. $570 \mathrm{p}$.

GASPAROTTO, F. et al. Eficiência de métodos para detecção de Didymella bryoniae associado a sementes de híbridos de meloeiros nobres. Acta Scientiarum Agronomy, Maringá, v. 31, n. 3, p. 397-402, 2009.

GIBSON, I. A. S. Saprophytic fungi as destroyers of germinating pine seeds. East African Agricultural Journal, Nairobi, [Sn.; S1.], p. 203-203, 1957.

LISBOA-PADULLA, T. et al. Tratamento de sementes de pau-brasil com fungicidas: efeito na incidência de fungos, germinação e transmissão de fungos pelas sementes. Summa Phytopathologica, Botucatu, v. 35, n. 2, p. 148-150, 2009.

LUCCA FILHO, O. A. Metodologia dos testes de sanidade de sementes. In: MENTEN, J. O. M. (Ed.). Patógenos em sementes: deteç̧ão, danos e controle químico. Piracicaba: Esalq, 1991. p. 276-298.

MACHADO, J. C. Patologia de sementes: fundamentos e aplicações. Brasília: Ministério da Educação: ESAL: FAEPE, 1988. 107 p.

MAGALHÃES, H. M. et al. Qualidade sanitária de sementes de coquinho-azedo (Butia capitata) no Norte de Minas. Ciência Rural, Santa Maria, v. 38, n. 8, p. 2371-2374, nov. 2008.

MEDEIROS, A. C. S. et al. Avaliação qualiquantitativa de fungos associados à sementes de aroeira (Astronium urundeuva (FR. ALL.) ENGL.). Revista Brasileira de Sementes, Brasília, v. 14, n. 1, p. 51-55, 1992.

MENDES, S. S. et al. Levantamento, patogenicidade e transmissão de fungos associados às sementes de sabiá (Mimosa caesalpiniaefolia Benth). Revista 
Ciência Agronômica, Fortaleza, v. 36, n. 1, p. 118122, jan./abr. 2005.

MIETH, A. T. Microflora e qualidade fisiológica de sementes de cedro (Cedrella fissilis) tratadas com extrato natural de hortelã (Mentha piperita). IN: CONGRESSO BRASILEIRO DE AGROECOLOGIA, 5., 2007, Guarapari. Anais... Guarapari: ABA, 2007.

MORENO, J. A. Clima do Rio Grande do Sul. Porto Alegre: Secretaria da Agricultura, 1961. 73 p. NECHET, K. L.; ABREU, M. S. Caracterização morfológica e testes de patogenicidade de isolados de Colletotrichum sp. obtidos de cafeeiro. Ciência e Agrotecnologia, Lavras, v. 26, n. 6, p. 1135-1142, nov./dez. 2002.

NEERGAARD, P. Seed pathology. London: Mac Millan Press, 1979. 829. p. v. 2.

OLIVEIRA, J. A. et al. Comportamento de sementes de milho tratadas com fungicidas antes e após o armazenamento convencional. Revista Brasileira de Sementes, Brasília, v. 19, n. 2, p. 208-213, 1997. POLETTO, I. et al. Primeira ocorrência de Pythium sp. e Rhizoctonia sp. causando podridões de raízes em ervais no Rio Grande do Sul. Ciência Florestal, Santa Maria, v. 17, n. 1, p. 65- 69, jan./mar. 2007.

REGO, S. S. Germinação, morfologia e sanidade de sementes de Blepharocalyx salicifolius (H.B.K.) Berg e Myrceugenia gertii Landrum - Myrtaceae. 2008. 114 f. Dissertação (Mestrado em Engenharia Florestal)-Universidade Federal do Paraná, Curitiba, 2008.

REITZ, R.; KLEIN, R. M.; REIS, A. Projeto madeira do Rio Grande do Sul. Itajaí: IOESC, $1983.525 \mathrm{p}$.

RUIZ FILHO, R. R. et al. Fungos associados às sementes de cedro. Summa Phytopathologica, Botucatu, v. 30, n. 4, p. 494-496, 2004.

SANTOS, A. F. dos; GRIGOLETTI JÚNIOR, A.; AUER, C. G. Transmissão de fungos por sementes de espécies florestais. Floresta, Curitiba, v. 30, n. 1/2, p. 119-128, 2000.

SANTOS, G. R. et al. Transporte, transmissibilidade e patogenicidade da microflora associada à sementes de feijão (Phaseolus vulgaris). Revista Ceres, Viçosa, v. 43, n. 249, p. 621-627, 1996.

SANTOS, G. R.; ARAÚJO, E.; BRUNO, R. L. A. Investigações preliminares sobre detecção e patogenicidade da microflora de sementes de urucu (Bixa orellana L.). Revista Brasileira de Sementes, Brasília, v. 14, n. 1, p. 13-15, 1992.

SARTORI, A. F.; REIS, E. M.; CASA, R. T. Quantificação da transmissão de Fusarium moniliforme de sementes para plântulas de milho. Fitopatologia Brasileira, Brasília, v. 29, n. 4, p. 456-458, jul./ago. 2004.

SILVEIRA, S. F. da. et al. Characterization of Rhizoctonia species associated with foliar necrosis and leaf scorch of clonally-propagated Eucalyptus in Brazil. European Journal of Plant Pathology, Holanda, v. 106, p. 27-36, 2000.

ZONTA, E. P.; MACHADO, A. A. Sistema de análise estatística para microcomputadores SANEST. Pelotas: UFPel, Instituto de Física e Matemática, 1986. $150 \mathrm{p}$. 